\title{
On Representation Theorem for Algebras with Three Commuting
} Involutions

Amir A. Mohammed

amirabdulillah64@gmail.com

\author{
Baida S. Abdullah Nadwa S. Younis
}

College of Education

University of Mosul, Iraq

\section{Received on: $26 / 08 / 2007$}

\section{ABSTRACT}

Accepted on: 30/01/2008

Algebras with three commuting involutions are represented as commutants of one-generated $\diamond-\square-\bigcirc$ subalgebras of algebras of vectorspace endomorphisms where $\diamond-\square$ and $\bigcirc$ are involutions of a prefixed type.

Keywords: Algebras, commuting involutions.

$$
\begin{aligned}
& \text { حول نظرية التمثيل للجبريات مع ثلاث تثابكات إبدالية }
\end{aligned}
$$

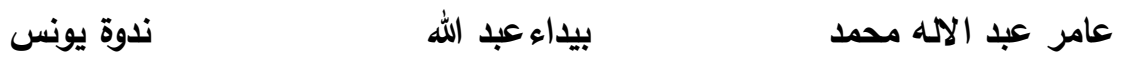

$$
\begin{aligned}
& \text { كلية التربية، جامعة الموصل } \\
& \text { تاريخ القبول: 2008/01/30 } \\
& \text { تاريخ الاستلام: 2007/08/26 } \\
& \text { الملخص }
\end{aligned}
$$

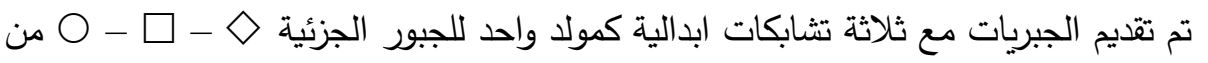

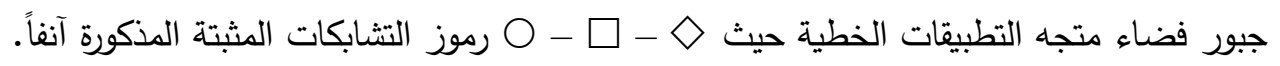

$$
\begin{aligned}
& \text { الكلمات المفتاحية: الجبريات، تثابكات ابدالية. }
\end{aligned}
$$

\section{Introduction and Preliminaries}

Throughout this paper $(k,-)$ denotes a field with an involution and the terminology of algebra and algebra involution is relative to $(k,-)$. A systematic study of representation theory for algebras with involutions was given in [6] by Quebbemann and he proved that (involutive unital finitedimensional algebras can be represented as commutants of one-generated self-adjoint subalgebras of algebras of vector-space endomorphisms) and later the representation theory for algebras with involutions has been extend to algebras with two commuting involutions by Cabrera and Mohammed see [1].

We begin by summarizing some definitions and fundamental concepts. An involution * in an algebra $A$ is a mapping $a \rightarrow a^{*}$ of $A$ into it self satisfying $(a+b)^{*}=a^{*}+b^{*},(\alpha a)^{*}=\bar{\alpha} \square a^{*}$ (where - denote the conjecate of complex number), $(a b)^{*}=b^{*} a^{*}$ and $a^{* *}=a$ for all $a, b$ in $A$ and $\alpha$ in $k$ see[1]. A subalgebra of $A$ globally invariant by $*$ is called a 
*-subalgebra. If $B$ is a $*$ - subalgebra of $A$, then its centralizer in $A$ given by

$\{a \in A: a b=$ ba for all $b$ in $B\}$,

is also a * subalgebra of $A$ see [1].

Involutive algebras can be constructed from the consideration of nondegenerate hermitian spaces. Recall that, for $\Sigma$ in $k$ satisfying $\Sigma \bar{\Sigma}=1$, a nondegenerate $\Sigma$-hermitian form in a vector - space $M$ over $k$ is a mapping $<.$, . > from $M \times M$ into $k$ satisfying

$\left\langle m_{1}+m_{2}, m^{\prime}\right\rangle=\left\langle m_{1}, m^{\prime}\right\rangle+\left\langle m_{2}, m^{\prime}\right\rangle,\left\langle\alpha m, m^{\prime}\right\rangle=\alpha\left\langle m, m^{\prime}\right\rangle$ $\left\langle m, m^{\prime}\right\rangle=\Sigma\left\langle\overline{m^{\prime}, m}\right\rangle$

for all $m_{1}, m_{2}, m, m^{\prime}$ in $M$ and $\alpha$ in $k$, and $\left\langle m, m^{\prime}\right\rangle=0$ for all $m^{\prime}$ implies $m=0$ see [1]. If $M$ has finite dimension, then the algebra $\operatorname{End}_{\mathrm{k}}(M)$ of all endomorphisms of $M$ with the adjoint involution $F \rightarrow F^{\diamond}$ given by

$\left\langle F(m), m^{\prime}\right\rangle=\left\langle m, F^{\diamond}\left(m^{\prime}\right)\right\rangle$

for all $m, m^{\prime}$ in $M$ see [1].

If $(A, *, \#, \delta),(B, \diamond, \square, O)$ are algebras with three commuting involutions, an isomorphism between $(A, *, \#, \delta)$ and $(B, \diamond, \square, O)$ is an algebra isomorphism $\phi$ from $A$ onto $B$ satisfying $\phi\left(a^{*}\right)=\phi(a)^{\diamond}, \phi\left(a^{\#}\right)=$ $\phi(a)^{G}$ and $\phi\left(a^{\delta}\right)=\phi(a)^{F}$ for all $a$ in $A$. In this case $(A, *, \#, \delta)$ and $(B, \diamond, \square$ , $O)$ are said to be isomorphic see [1].

Our main result is the following :

Theorem 1. Let $(A, *, \#, \delta)$ be a unital finite-dimensional algebra with commuting involutions over $(k,-)$ and let $\Sigma, \Sigma^{\prime}, \Sigma^{\prime \prime}$ in $k$ such that $\Sigma \bar{\Sigma}=\Sigma^{\prime} \bar{\Sigma}^{\prime}$ $=\Sigma^{\prime} \bar{\Sigma}^{\prime \prime}=1$. Then there exist a finite-dimensional vector space $w$ over $k$, a nondegenerate $\Sigma$-hermitian form <. , .>, a nondegenerate $\Sigma$ '-hermitian form [. , .], a nondegenerate $\Sigma$ "-hermitian form [. , .], and $F$ in $\operatorname{End}_{\mathrm{k}}(w)$ such that $(A, *, \#, \delta)$ is isomorphic to the centralizer of the $\diamond-\square-O-$ subalgebra of $\operatorname{End}_{\mathrm{k}}(w)$ generated by $F$, where $\diamond, \square$ and $O$ are adjoint involutions in $\operatorname{End}_{\mathrm{k}}(w)$ determined by $<., .>,[.,$.$] and (.,$.$) , respectively.$

We will follow the lines of the following lemma : Let $(A, *, \#)$ be a unital finite-dimensional algebra with commuting involutions over $(K,-)$ and let $\Sigma, \Sigma^{\prime}$ in $K$ such that $\Sigma \bar{\Sigma}=\Sigma^{\prime} \bar{\Sigma}^{\prime}=1$. Then there exist a finitedimensional vector space $W$ over $K$, a nondegenerate $\Sigma$-hermitian form <.,. > in $W$, a nodegenerate $\Sigma^{\prime}$-hermitian from [. . .] in $W$, and $F$ in $\operatorname{End}_{K}(W)$ such that $(A, *, \#)$ is isomorphic to the centralizer of the $\diamond-\square$ - subalgebra of $\operatorname{End}_{K}(W)$ generated by $F$, where $\diamond$ and $\square$ are adjoint involutions in $\operatorname{End}_{K}(W)$ determined by $<.$, . > and [. , .] respectively.

Proof : see [1, Theorem 1] 
The first part of the proof consists in finding a nondegenerate $\Sigma-\Sigma^{\prime}-\Sigma^{\prime \prime}$-hermitian space $w_{o}$ over $k$ such that $(A, *, \#, \delta)$ is embedded into $\operatorname{End}_{\mathrm{k}}\left(w_{o}\right)$ in such a way that $w_{o}$ is a balanced A-module (that is, $A=$ End $_{\mathrm{B}}$ $\left(w_{o}\right)$ if $\left.B=\operatorname{End}_{A}\left(w_{o}\right)\right)$. Our construction involves the three commuting involutions of $A$ and consists in a convenient triple of the representation used in [1].

Theorem 2. let $(A, *, \#, \delta)$ be a unital finite-dimensional algebra with commuting involutions over $(k,-)$ and let $\Sigma, \Sigma^{\prime}, \Sigma^{\prime \prime}$ in $k$ such that $\Sigma \bar{\Sigma}=\Sigma \bar{\Sigma}^{\prime}$ $=\Sigma^{\prime \prime} \bar{\Sigma}^{\prime \prime}=1$. Then there exists $\left(w_{o},<., .>,[.,],.(.,).\right)$, where $w_{o}$ is a finite-dimensional vector space over $k$ which is a balanced left $A$-module (in fact, $w_{o}$ contains $A$ as a direct summand) and <. , .> , [. , .] , $(.,$.$) are nondegenerate \Sigma$-hermitian, $\Sigma$ '-hermitian and $\Sigma$ "-hermitian forms in $w_{o}$, respectively, in such a way that the associated representation of $A$ in $w_{o}$ becomes an isomorphism of algebras with three involutions of $(A, *, \#, \delta)$ into $\left(\operatorname{End}_{\mathrm{k}}\left(w_{o}\right), \diamond, \square, O\right)$, where $\diamond, \square$ and $\bigcirc$ are adjoint involutions in $\operatorname{End}_{\mathrm{k}}\left(w_{o}\right)$ determined by <., .> , [., . .] and (., . .), respectively.

Proof. Consider the vector space $\mathrm{w}_{\mathrm{o}}:=U_{1} \oplus U_{2} \oplus U_{3} \oplus U_{4} \oplus U_{5} \oplus U_{6}$, where $U_{1}=U_{3}=U_{5}=A$ and $U_{2}=U_{4}=U_{6}=\operatorname{Hom}_{k}(A, k)$. Endow $w_{o}$ with the structure of faithful left $A$-module given by :

$a\left(x_{1}, f_{1}, x_{2}, f_{2}, x_{3}, f_{3}\right):=\left(a x_{1}, f_{1} o L_{a^{*}}, a^{* \#} x_{2}, f_{2} o L_{a \#,} a^{* \# \delta} x_{3}, f_{3} o L_{a \delta}\right)$ for all $a$ in $A$ and $\left(x_{1}, f_{1}, x_{2}, f_{2}, x_{3}, f_{3}\right)$ in $w_{o}$. The mapping <., .> from $w_{o} \times w_{o}$ in to $k$ defined by

$<\left(x_{1}, f_{1}, x_{2}, f_{2}, x_{3}, f_{3}\right),\left(y_{1}, g_{1}, y_{2}, g_{2}, y_{3}, g_{3}\right)>:=$
$f_{1}\left(y_{1}\right)+f_{2}\left(y_{2}\right)+f_{3}\left(y_{3}\right)+\Sigma\left(g_{1}\left(x_{1}\right)+g_{2}\left(x_{2}\right)+g_{3}\left(x_{3}\right)\right)$

is a nondegenerate $\Sigma$-hermitian form satisfying

$\left\langle a\left(x_{1}, f_{1}, x_{2}, f_{2}, x_{3}, f_{3}\right),\left(y_{1}, g_{1}, y_{2}, g_{2}, y_{3}, g_{3}\right)\right\rangle=$

$\left\langle\left(x_{1}, f_{1}, x_{2}, f_{2}, x_{3}, f_{3}\right), a^{*}\left(y_{1}, g_{1}, y_{2}, g_{2}, y_{3}, g_{3}\right)>\right.$,

and therefore the representation of $A$ on $w_{o}$ becomes an isomorphism of involutive algebras from $(A, *)$ into $\left(\operatorname{End}_{\mathrm{k}}\left(w_{o}\right), \diamond\right)$, where $\diamond$ denotes the adjoint involution with respect to $<.$, . $>$. Furthermore, the mapping [. , .] from $w_{o} \times w_{o}$ into $\mathrm{k}$ defined by

$\left[\left(x_{1}, f_{1}, x_{2}, f_{2}, x_{3}, f_{3}\right),\left(y_{1}, g_{1}, y_{2}, g_{2}, y_{3}, g_{3}\right)\right]:=$ $\overline{f_{1}\left(y_{2}\right)+f_{2}\left(y_{3}\right)+f_{3}\left(y_{1}\right)}+\Sigma^{\prime}\left(g_{1}\left(x_{2}\right)+g_{2}\left(x_{3}\right)+g_{3}\left(x_{1}\right)\right)$

is a nondegenerate $\Sigma^{\prime}$-hermitian form satisfying

$\left[a\left(x_{1}, f_{1}, x_{2}, f_{2}, x_{3}, f_{3}\right),\left(y_{1}, g_{1}, y_{2}, g_{2}, y_{3}, g_{3}\right)\right]=$ $\left[\left(x_{1}, f_{1}, x_{2}, f_{2}, x_{3}, f_{3}\right), a^{\#}\left(y_{1}, g_{1}, y_{2}, g_{2}, y_{3}, g_{3}\right)\right]$, 
and so the representation of $A$ on $w_{o}$ also becomes an isomorphism of involutive algebras from $(A, \#)$ into $\left(\operatorname{End}_{\mathrm{k}}\left(w_{o}\right), \boldsymbol{G}\right)$, where $\boldsymbol{G}$ denotes the adjoint involution with respect to [.,.]. furthermore, the mapping (. , .) from $w_{o} \mathrm{x} w_{o}$ into k defined by

$\left(\left(x_{1}, f_{1}, x_{2}, f_{2}, x_{3}, f_{3}\right),\left(y_{1}, g_{1}, y_{2}, g_{2}, y_{3}, g_{3}\right)\right):=$

$\overline{f_{1}\left(y_{3}\right)+f_{2}\left(y_{1}\right)+f_{3}\left(y_{2}\right)}+\Sigma^{\prime \prime}\left(g_{1}\left(x_{3}\right)+g_{2}\left(x_{1}\right)+g_{3}\left(x_{2}\right)\right)$

is a nondegenerate $\Sigma$ "-hermitian form satisfying

$\left[a\left(x_{1}, f_{1}, x_{2}, f_{2}, x_{3}, f_{3}\right),\left(y_{1}, g_{1}, y_{2}, g_{2}, y_{3}, g_{3}\right)\right]=$

$\left[\left(x_{1}, f_{1}, x_{2}, f_{2}, x_{3}, f_{3}\right), a^{\delta}\left(y_{1}, g_{1}, y_{2}, g_{2}, y_{3}, g_{3}\right)\right]$,

and so the representation of $A$ on $w_{o}$ also becomes an isomorphism of involutive algebras from $(A, \delta)$ into $\left(\operatorname{End}_{\mathrm{k}}\left(w_{o}\right), \boldsymbol{F}\right)$, where $\boldsymbol{F}$ denotes the adjoint involution with respect to (., .). Therefore, the representation of $A$ on $w_{o}$ is an isomorphism of algebras with three involutions. Since $w_{o}$ contains the "regular" $A$-module $A$ as a direct summand, it is balanced (see [4, P. 451]).

Remark 1. The involutions $\diamond, \square$ and $O$ in $\operatorname{End}_{\mathrm{k}}\left(w_{o}\right)$ obtained in the above proof are not necessarily commuting. Since $w_{o}=U_{1} \oplus U_{2} \oplus U_{3} \oplus U_{4} \oplus U_{5}$ $\oplus U_{6}$ we can represent each $T$ in End $_{\mathrm{k}}\left(w_{o}\right)$ as a $6 \times 6$ homomorphism matrix.

$$
T=\left(\begin{array}{llllll}
T_{11} & T_{12} & T_{13} & T_{14} & T_{15} & T_{16} \\
T_{21} & T_{22} & T_{23} & T_{24} & T_{25} & T_{26} \\
T_{31} & T_{32} & T_{33} & T_{34} & T_{35} & T_{36} \\
T_{41} & T_{42} & T_{43} & T_{44} & T_{45} & T_{46} \\
T_{51} & T_{52} & T_{53} & T_{54} & T_{55} & T_{56} \\
T_{61} & T_{62} & T_{63} & T_{64} & T_{65} & T_{66}
\end{array}\right)
$$

Where $T_{i j} \in \operatorname{Hom}_{k}\left(U_{j}, U_{i}\right)$ for $i, j \in\{1,2,3,4,5,6\}$. It is easy to verify that

$$
T^{\diamond}=\left(\begin{array}{cccccc}
T_{22}^{\prime} & \bar{\Sigma} T_{12}^{\prime} & T_{42}^{\prime} & \bar{\Sigma} T_{32}^{\prime} & T_{62}^{\prime} & \bar{\Sigma} T_{52}^{\prime} \\
\Sigma T_{21}^{\prime} & T_{11}^{\prime} & \Sigma T_{41}^{\prime} & T_{31}^{\prime} & \Sigma T_{61}^{\prime} & T_{51}^{\prime} \\
T_{24}^{\prime} & \bar{\Sigma} T_{14}^{\prime} & T_{44}^{\prime} & \bar{\Sigma} T_{34}^{\prime} & T_{64}^{\prime} & \bar{\Sigma} T_{54}^{\prime} \\
\Sigma T_{23}^{\prime} & T_{13}^{\prime} & \Sigma T_{43}^{\prime} & T_{33}^{\prime} & \Sigma T_{63}^{\prime} & T_{53}^{\prime} \\
T_{26}^{\prime} & \bar{\Sigma} T_{16}^{\prime} & T_{46}^{\prime} & \bar{\Sigma} T_{36}^{\prime} & T_{66}^{\prime} & \bar{\Sigma} T_{56}^{\prime} \\
\Sigma T_{25}^{\prime} & T_{15}^{\prime} & \Sigma T_{45}^{\prime} & T_{35}^{\prime} & \Sigma T_{65}^{\prime} & T_{55}^{\prime}
\end{array}\right),
$$


$T^{\square}=\left(\begin{array}{cccccc}T_{66}^{\prime} & \overline{\Sigma^{\prime}} T_{56}^{\prime} & T_{46}^{\prime} & \overline{\Sigma^{\prime}} T_{36}^{\prime} & T_{26}^{\prime} & \overline{\Sigma^{\prime}} T_{16}^{\prime} \\ \Sigma^{\prime} T_{65}^{\prime} & T_{55}^{\prime} & \Sigma^{\prime} T_{45}^{\prime} & T_{35}^{\prime} & \Sigma^{\prime} T_{25}^{\prime} & T_{15}^{\prime} \\ T_{64}^{\prime} & \overline{\Sigma^{\prime}} T_{54}^{\prime} & T_{44}^{\prime} & \overline{\Sigma^{\prime}} T_{34}^{\prime} & T_{24}^{\prime} & \overline{\Sigma^{\prime}} T_{14}^{\prime} \\ \Sigma^{\prime} T_{63}^{\prime} & T_{53}^{\prime} & \Sigma^{\prime} T_{43}^{\prime} & T_{33}^{\prime} & \Sigma^{\prime} T_{23}^{\prime} & T_{13}^{\prime} \\ T_{62}^{\prime} & \overline{\Sigma^{\prime}} T_{52}^{\prime} & T_{42}^{\prime} & \overline{\Sigma^{\prime}} T_{32}^{\prime} & T_{22}^{\prime} & \overline{\Sigma^{\prime}} T_{12}^{\prime} \\ \Sigma^{\prime} T_{61}^{\prime} & T_{51}^{\prime} & \Sigma^{\prime} T_{41}^{\prime} & T_{31}^{\prime} & \Sigma^{\prime} T_{21}^{\prime} & T_{11}^{\prime}\end{array}\right)$

And

$T^{O}=\left(\begin{array}{cccccc}T_{44}^{\prime} & \overline{\Sigma^{\prime \prime}} T_{14}^{\prime} & T_{64}^{\prime} & \overline{\Sigma^{\prime \prime}} T_{34}^{\prime} & T_{24}^{\prime} & \overline{\Sigma^{\prime \prime}} T_{54}^{\prime} \\ \Sigma^{\prime \prime} T_{41}^{\prime} & T_{11}^{\prime} & \Sigma^{\prime} T_{61}^{\prime} & T_{31}^{\prime} & \Sigma^{\prime \prime} T_{21}^{\prime} & T_{51}^{\prime} \\ T_{46}^{\prime} & \overline{\Sigma^{\prime \prime}} T_{16}^{\prime} & T_{66}^{\prime} & \overline{\Sigma^{\prime \prime}} T_{36}^{\prime} & T_{26}^{\prime} & \overline{\Sigma^{\prime \prime}} T_{56}^{\prime} \\ \Sigma^{\prime \prime} T_{43}^{\prime} & T_{13}^{\prime} & \Sigma^{\prime \prime} T_{63}^{\prime} & T_{33}^{\prime} & \Sigma^{\prime \prime} T_{23}^{\prime} & T_{53}^{\prime} \\ T_{42}^{\prime} & \overline{\Sigma^{\prime \prime}} T_{12}^{\prime} & T_{62}^{\prime} & \overline{\Sigma^{\prime \prime}} T_{32}^{\prime} & T_{22}^{\prime} & \overline{\Sigma^{\prime \prime}} T_{52}^{\prime} \\ \Sigma^{\prime \prime} T_{45}^{\prime} & T_{15}^{\prime} & \Sigma^{\prime \prime} T_{65}^{\prime} & T_{35}^{\prime} & \Sigma^{\prime \prime} T_{25}^{\prime} & T_{55}^{\prime}\end{array}\right)$

Therefore

$$
\begin{aligned}
& T^{\diamond \square}=\left(\begin{array}{cccccc}
T_{55}^{\prime} & \overline{\Sigma^{\prime}} \Sigma T_{56}^{\prime} & T_{53}^{\prime} & \overline{\Sigma^{\prime}} \Sigma T_{54}^{\prime} & T_{51}^{\prime} & \overline{\Sigma^{\prime}} \Sigma T_{52}^{\prime} \\
\Sigma^{\prime} \bar{\Sigma} T_{65}^{\prime} & T_{66}^{\prime} & \Sigma^{\prime} \bar{\Sigma} T_{63}^{\prime} & T_{64}^{\prime} & \Sigma^{\prime} \bar{\Sigma} T_{61}^{\prime} & T_{62}^{\prime} \\
T_{35}^{\prime} & \overline{\Sigma^{\prime}} \Sigma T_{36}^{\prime} & T_{33}^{\prime} & \overline{\Sigma^{\prime}} \Sigma T_{34}^{\prime} & T_{31}^{\prime} & \overline{\Sigma^{\prime}} \Sigma T_{32}^{\prime} \\
\Sigma^{\prime} \bar{\Sigma} T_{45}^{\prime} & T_{46}^{\prime} & \Sigma^{\prime} \bar{\Sigma} T_{43}^{\prime} & T_{44}^{\prime} & \Sigma^{\prime} \bar{\Sigma} T_{41}^{\prime} & T_{42}^{\prime} \\
T_{15}^{\prime} & \overline{\Sigma^{\prime}} \Sigma T_{16}^{\prime} & T_{13}^{\prime} & \overline{\Sigma^{\prime}} \Sigma T_{14}^{\prime} & T_{11}^{\prime} & \overline{\Sigma^{\prime}} \Sigma T_{12}^{\prime} \\
\Sigma^{\prime} \bar{\Sigma} T_{25}^{\prime} & T_{26}^{\prime} & \Sigma^{\prime} \bar{\Sigma} T_{23}^{\prime} & T_{24}^{\prime} & \Sigma^{\prime} \bar{\Sigma} T_{21}^{\prime} & T_{22}^{\prime}
\end{array}\right) \\
& T^{\square \diamond}=\left(\begin{array}{cccccc}
T_{55}^{\prime} & \bar{\Sigma} \Sigma^{\prime} T_{56}^{\prime} & T_{53}^{\prime} & \bar{\Sigma} \Sigma^{\prime} T_{54}^{\prime} & T_{51}^{\prime} & \bar{\Sigma} \Sigma^{\prime} T_{52}^{\prime} \\
\Sigma \overline{\Sigma^{\prime}} T_{65}^{\prime} & T_{66}^{\prime} & \Sigma \overline{\Sigma^{\prime}} T_{63}^{\prime} & T_{64}^{\prime} & \Sigma \overline{\Sigma^{\prime}} T_{61}^{\prime} & T_{62}^{\prime} \\
T_{35}^{\prime} & \bar{\Sigma} \Sigma^{\prime} T_{36}^{\prime} & T_{33}^{\prime} & \bar{\Sigma} \Sigma^{\prime} T_{34}^{\prime} & T_{31}^{\prime} & \bar{\Sigma} \Sigma^{\prime} T_{32}^{\prime} \\
\Sigma \overline{\Sigma^{\prime}} T_{45}^{\prime} & T_{46}^{\prime} & \Sigma \overline{\Sigma^{\prime}} T_{43}^{\prime} & T_{44}^{\prime} & \Sigma \overline{\Sigma^{\prime}} T_{41}^{\prime} & T_{42}^{\prime} \\
T_{15}^{\prime} & \bar{\Sigma} \Sigma^{\prime} T_{16}^{\prime} & T_{13}^{\prime} & \bar{\Sigma} \Sigma^{\prime} T_{14}^{\prime} & T_{11}^{\prime} & \bar{\Sigma} \Sigma^{\prime} T_{12}^{\prime} \\
\Sigma \overline{\Sigma^{\prime}} T_{25}^{\prime} & T_{26}^{\prime} & \Sigma \overline{\Sigma^{\prime}} T_{23}^{\prime} & T_{24}^{\prime} & \Sigma \overline{\Sigma^{\prime}} T_{21}^{\prime} & T_{22}^{\prime}
\end{array}\right),
\end{aligned}
$$

And 


$$
T^{\diamond 0}=\left(\begin{array}{cccccc}
T_{55}^{\prime} & \overline{\Sigma^{\prime \prime}} \Sigma T_{56}^{\prime} & T_{53}^{\prime} & \overline{\Sigma^{\prime \prime}} \Sigma T_{54}^{\prime} & T_{51}^{\prime} & \overline{\Sigma^{\prime \prime}} \Sigma T_{52}^{\prime} \\
\Sigma^{\prime \prime} \bar{\Sigma} T_{65}^{\prime} & T_{66}^{\prime} & \bar{\Sigma}^{\prime \prime} \bar{\Sigma} T_{63}^{\prime} & T_{64}^{\prime} & \Sigma^{\prime \prime} \bar{\Sigma} T_{61}^{\prime} & T_{62}^{\prime} \\
T_{35}^{\prime} & \overline{\Sigma^{\prime \prime}} \Sigma T_{36}^{\prime} & T_{33}^{\prime} & \overline{\Sigma^{\prime \prime}} \Sigma T_{34}^{\prime} & T_{31}^{\prime} & \overline{\Sigma^{\prime \prime}} \Sigma T_{32}^{\prime} \\
\Sigma^{\prime \prime} \bar{\Sigma} T_{45}^{\prime} & T_{46}^{\prime} & \Sigma^{\prime \prime} \bar{\Sigma} T_{43}^{\prime} & T_{44}^{\prime} & \Sigma^{\prime \prime} \bar{\Sigma} T_{41}^{\prime} & T_{42}^{\prime} \\
T_{15}^{\prime} & \overline{\Sigma^{\prime \prime}} \Sigma T_{16}^{\prime} & T_{13}^{\prime} & \overline{\Sigma^{\prime \prime}} \Sigma T_{14}^{\prime} & T_{11}^{\prime} & \overline{\Sigma^{\prime \prime}} \Sigma T_{12}^{\prime} \\
\Sigma^{\prime \prime} \bar{\Sigma} T_{25}^{\prime} & T_{26}^{\prime} & \Sigma^{\prime \prime} \bar{\Sigma} T_{23}^{\prime} & T_{24}^{\prime} & \Sigma^{\prime \prime} \bar{\Sigma} T_{21}^{\prime} & T_{22}^{\prime}
\end{array}\right)
$$

As a result, $\diamond, \square$ and $\bigcirc$ are commuting if and only if $\overline{\Sigma^{\prime}} \Sigma=\bar{\Sigma} \Sigma^{\prime}=\overline{\Sigma^{\prime \prime}} \Sigma=$ $\bar{\Sigma} \Sigma^{\prime \prime}$, or equivalently $\Sigma^{2}=\Sigma^{2}=\Sigma^{\prime \prime 2}$.

Proof of Theorem 1. let $(A, *, \#, \delta)$ be a unital finite-dimensional algebra with commuting involutions over $(k,-)$ and let $\Sigma, \Sigma, \Sigma^{\prime \prime}$ in $k$ such that $\Sigma \bar{\Sigma}=\Sigma^{\prime} \overline{\Sigma^{\prime}}=\Sigma^{\prime \prime} \overline{\Sigma^{\prime \prime}}=1$. By Theorem 2 there exists $\left(w_{o},<_{., .}>,[.,],.(.,).\right)$, where $\mathrm{w}_{\mathrm{o}}$ is a finite-dimensional vector space over $\mathrm{k}$ which is a balanced left A-module and <.,.>, [.,.] and (.,.) are nondegenerate $\Sigma$-hermitian, $\Sigma^{\prime}$ hermitian and $\Sigma^{\prime \prime}$-hermitian forms in $w_{o}$, respectively, in such a way the associated representation of $A$ in $w_{o}$ becomes an isomorphism from $(A, *$, \#, $\delta$ ) into $\left(\operatorname{End}_{\mathrm{k}}\left(w_{o}\right), \diamond, \square, O\right)$, where $\diamond, \square$ and $\bigcirc$ are adjoint involutions in End $_{\mathrm{k}}\left(w_{o}\right)$ determined by <.,.>, [.,.] and (.,.), respectively. Let $\mathrm{m}$ denote the dimension of $B=\operatorname{End}_{\mathrm{A}}\left(w_{o}\right)$. Put $(w,<., .>,[.,],.(.,)):.=\left(w_{o},<_{., .}\right)$, , [...] , (.,.)) $\oplus$ m...... $\oplus\left(w_{o},<., .>,[.,],.(.,).\right)$, and consider $\operatorname{End}_{\mathrm{k}}\left(w_{o}\right)$ embedded diagonally in $\operatorname{End}_{\mathrm{k}}(w)$. By the final step of the proof of theorem 1 in [1] applied to $<_{., .}>,[.,$.$] and (...) there exists \mathrm{F}$ in $\operatorname{End}_{\mathrm{k}}(w)$ such that $\mathrm{A}=\operatorname{End}_{\mathrm{C}}(w)$ $=\operatorname{End}_{\mathrm{D}}(w)=\operatorname{End}_{\mathrm{H}}(w)$, where $C$ and $D$ (resp. $\left.H\right)$ denotes the $\nabla-$ subalgebra and $\square$-subalgebra (resp. $O$ - subalgebra) of $\operatorname{End}_{\mathrm{K}}(w)$ generated by $F$. Let us denote by $\mathrm{E}$ the $\checkmark-\square-O$ - subalgebra of $\operatorname{End}_{\mathrm{k}}(w)$ generated by $F$. Since $C, D, H \subseteq E$, it follows that $\operatorname{End}_{\mathrm{E}}(w) \subseteq \operatorname{End}_{\mathrm{C}}(w)=\operatorname{End}_{\mathrm{D}}(w)=\operatorname{End}_{\mathrm{H}}(w)$ $=A$. On the other hand, $A$ is a $\searrow-\square-O-$ subalgebra of $\operatorname{End}_{\mathrm{k}}(w)$ whose elements commute with $F$, therefore $\operatorname{End}_{\mathrm{A}}(w)$ is a $\checkmark-\square-O$ - subalgebra of $\operatorname{End}_{\mathrm{k}}(w)$ containing $F$, and so $E \subseteq \operatorname{End}_{\mathrm{A}}(w)$. From this, $A \subseteq \operatorname{End}_{\mathrm{E}}(w)$. 
Remark 2. The process of representing the algebras of commuting involutions can be explained through the following diagram :

\begin{tabular}{|c|c|c|c|c|}
\hline $\begin{array}{c}\text { No. of } \\
\text { involution }\end{array}$ & $\begin{array}{c}\text { Underliny vector - } \\
\text { space of represented } \\
\text { algebra }\end{array}$ & $\begin{array}{c}\text { Construction of } \\
\text { Nondegenerate form } \\
\text { with respect to } \\
\text { involution } \\
\end{array}$ & $\begin{array}{c}\text { Generator of } \\
\text { represented } \\
\text { algebra with } \\
\text { involution } \\
\end{array}$ & 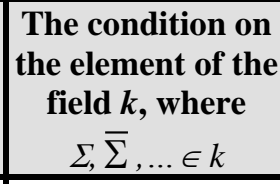 \\
\hline$N=1$ & $w_{o}=U_{1} \oplus U_{2}$ & $\langle.,\rangle$. & $\mid \begin{array}{c}\mathrm{T} \in \operatorname{End}_{\mathrm{k}}\left(\mathrm{w}_{\mathrm{o}}\right) \\
\text { and } \mathrm{T} \in \mathrm{M}_{2 \times 2}(\phi)\end{array}$ & $\Sigma \bar{\Sigma}=1$ \\
\hline$N=2$ & $w_{o}=U_{l} \oplus U_{2} \oplus U_{3} \oplus U_{4}$ & $<., .>,[.,]$. & $\left|\begin{array}{c}\mathrm{T} \in \operatorname{End}_{\mathrm{k}}\left(\mathrm{w}_{\mathrm{o}}\right) \\
\text { and } \mathrm{T} \in \mathrm{M}_{4 \times 4}(\phi)\end{array}\right|$ & $\Sigma \bar{\Sigma}=\Sigma^{\prime} \bar{\Sigma}^{\prime}=1$ \\
\hline$N=3$ & $w_{o}=U_{l} \oplus \ldots \ldots . \oplus U_{6}$ & $<., .>,[.,],.(.,)$. & $\begin{array}{c}\mathrm{T} \in \operatorname{End}_{\mathrm{k}}\left(\mathrm{w}_{\mathrm{o}}\right) \\
\text { and } \mathrm{T} \in \mathrm{M}_{6 \mathrm{x}}(\phi)\end{array}$ & $\begin{array}{c}\Sigma \bar{\Sigma}=\Sigma^{\prime} \bar{\Sigma}^{\prime}= \\
\Sigma^{\prime \prime} \overline{\Sigma^{\prime \prime}}=1\end{array}$ \\
\hline$N=4$ & $w_{o}=U_{1} \oplus \ldots \ldots . \oplus U_{8}$ & $<., .>,[.,],.(.,),.\{.,\}$. & $\begin{array}{c}\mathrm{T} \in \operatorname{End}_{\mathrm{k}}\left(\mathrm{w}_{\mathrm{o}}\right) \\
\text { and } \mathrm{T} \in \mathrm{M}_{8 \times 8}(\phi)\end{array}$ & $\begin{array}{c}\Sigma \bar{\Sigma}=\Sigma^{\prime} \overline{\Sigma^{\prime}}= \\
\Sigma^{\prime \prime} \overline{\Sigma^{\prime \prime}}=\Sigma^{\prime \prime \prime} \overline{\Sigma^{\prime \prime \prime}}=1\end{array}$ \\
\hline$N=5$ & $w_{o}=U_{1} \oplus \ldots \ldots . \oplus U_{10}$ & $\begin{array}{c}\langle.,\rangle,[., .],(., .),\{., .\}, \\
((., .))\end{array}$ & $\begin{array}{c}\mathrm{T} \in \operatorname{End}_{\mathrm{k}}\left(\mathrm{w}_{\mathrm{o}}\right) \\
\text { and } \mathrm{T} \in \mathrm{M}_{10 \times 10}(\phi)\end{array}$ & $\begin{array}{c}\Sigma \overline{\bar{\Sigma}}=\Sigma^{\prime} \overline{\bar{\Sigma}^{\prime}}= \\
\Sigma^{\prime \prime} \overline{\Sigma^{\prime \prime}}=\Sigma^{\prime \prime \prime} \overline{\bar{\Sigma}^{\prime \prime \prime}}= \\
\Sigma^{\prime \prime \prime} \overline{\Sigma^{\prime \prime \prime \prime}}=1\end{array}$ \\
\hline $\begin{array}{l}\cdot \\
\dot{.} \\
\dot{ }\end{array}$ & $\dot{\cdot} \cdot$ & • & $\begin{array}{l}\cdot \\
\cdot \\
.\end{array}$ & $\begin{array}{l}\cdot \\
\dot{ } \\
\dot{ }\end{array}$ \\
\hline
\end{tabular}




\section{REFERENCES}

[1] M. Cabrera and Amir A. Mohammed, "A representation theorem for algebras with commuting involutions", (2000), Linear algebra and its Applications 306 (25-31).

[2] M. Cabrera, A. Moreno, A. Rodriguez, E. I. zel'manov, "Jordan Polynomials can be analytically recognized," (1996), Studia Math, 137-147.

[3] N. Jacobson, "Lectures in abstract algebra II, Linear algebra", (1953), Grad, Texts in Mathematics, Vol., 31, Springer, New York.

[4] S. Lang, "Algebra, third edition", (1971), Addison-Wesley, New York.

[5] A. Moreno, "Extending the norm from special Jordan triple systems to their associative envelopes", Banach Algebras '97, Proc. 13th Internat. Conf. on Banach Algebras, July 20-August 3, 1997, Walter de gruyter, Berlin, 1998, pp. 363-375.

[6] H. G. Quebbemann, "A representation theorem for algebras with involution", linear algebra Appl., (1987), 193-195. 